\title{
Kriterien für eine Risiko-orientierte Probenahme nach der Verordnung (EG) Nr. 882/2004 und der AVV Rahmen-Überwachung
}

\author{
Dr. C. Berg \\ Veterinärwesen und Lebensmittelüberwachung, Goethestraße 12, 3330 Gütersloh
}

\section{Zusätzliche Informationen/Anlagen}

Ellerbroek, L. (2005) Fleischwirtschaft 12.

Berg, C. (2006) Fleischwirtschaft 1:21-25.

\section{Zusammenfassung/Erläuterung}

Zu den amtlichen Kontrolltätigkeiten gehören auch die Probenahme und -analysen im Lebensmittel- und Futtermittelbereich. Sie sind ab dem 01. Januar 2006 nach Artikel 3 Abs. 1 der Verordnung (EG) Nr. 882/2004 (Amtliche Kontrollen zur Überprüfung der Einhaltung des Lebensmittel- und Futtermittelrechts sowie der Bestimmungen über Tiergesundheit und Tierschutz (sog. Kontrollverordnung)) regelmäßig und auf der Grundlage eines Risiko-basierten Konzeptes mit angemessener Häufigkeit durchzuführen. Die Probenahme soll zudem nach $\S 9$ Abs. 1 der Allgemeinen Verwaltungsvorschrift Rahmenüberwachung (AVV RÜb) vorrangig beim Hersteller oder Importeur erfolgen. Die Prüfung der Produkte auf nachfolgenden Handelsstufen kann dabei auf solche Parameter beschränkt werden, die sich nach Verlassen des Herstellerbetriebes infolge des Transportes, der Lagerung oder weiteres Inverkehrbringen verändern können.

Vom Kreis Gütersloh wurde in den vergangenen Jahren im Rahmen eines Pilotprojektes im Regierungsbezirk Ostwestfalen ein Konzept zur Ermittlung von Planprobenzahlen erarbeitet. Dieses Konzept basiert auf den nach Artikel 3 Abs. 1 der Kontrollverordnung genannten Kriterien (siehe auch Abschlussbericht LAGV-Projektgruppe „Risiko-orientierte Probenahme“, 2005).
Ausgehend von dem „Risikoprofil“ der einzelnen Lebensmittel (Ellerbroek, 2005) kann die Planprobenzahl betriebsspezifisch anhand der Produktionsmenge und der Beurteilung des Eigenkontrollsystems (Beurteilungssystem nach Anlage 2 AVV RÜb Änderungsentwurf) ermittelt werden. Bei Einzelhandelsbetrieben mit eigener Herstellung (handwerklich strukturierte Betriebe) lässt sich die Probenzahl aus der Risiko-orientiert ermittelten Kontrollfrequenz ableiten.

Um täuschungsschutzrechtliche Aspekte bei der Probenahme und -untersuchung in ausreichendem Maße zu berücksichtigen, sind auch für diesen Bereich entsprechende Kriterien zur Ermittlung der Probenzahl festzulegen. Die Anzahl und Art der zu entnehmenden Proben richtet sich somit nach der Gewerbestruktur („Betriebs- und Produktionsprofil“), wie sie im Bereich der für die Probenahme zuständigen Behörde vorliegt.

Die Anwendung des Konzeptes und die damit verbundenen Auswirkungen auf die Probenahme werden anhand verschiedener Beispielbetriebe dargestellt.

\section{Diskussionsergebnis}

Eine Risiko-basierte Probenahme und Untersuchung von Produkten wird als sinnvolles Instrument zur Steigerung von Effizienz und Effektivität der amtlichen Kontrolle angesehen.

Die von Berg, Roth/Renz und Ellerbroek vorgeschlagenen Modelle bzw. Risikokategorisierung stellen wesentliche Aspekte der Risiko-basierten Probenahme und Untersuchung dar. Der ALTS spricht sich für die Erweiterung der Risiko-basierten Probenahme und Untersuchung um die Fragestellungen des Täuschungsschutzes als essentiellen Bestandteil des Probenmanagements aus. 